\title{
Major vascular trauma
}

\author{
Kirrily -Rae J. Warren ${ }^{1} \cdot$ Zsolt J. Balogh ${ }^{1}$
}

Published online: 6 December 2019

(c) Springer-Verlag GmbH Germany, part of Springer Nature 2019

This edition sees the publication of six papers focusing on the evolving management of major vascular injuries. Truncal vascular injuries remain relatively uncommon but are associated with disproportionately high morbidity and mortality. The growth and development of vascular and endovascular surgery have led to new ways to approach major vessel injury and blur even further the evidence from sporadic cases. As with most uncommon injuries, the best approach to management remains an area of active study with imperfect data available. To date, there remain more questions than clear answers when it comes to optimal management of vascular injuries - these papers highlight some of the current controversies and add to the growing body of literature.

Thoracic aortic injury remains a leading cause of immediate death following significant deceleration injury. By including those patients for whom thoracic injury was diagnosed at autopsy in their study, Trlica and colleagues remind us that only a small fraction of patients with partial or complete contained rupture of the aorta survive to hospital (36/175 in their series) (1). For those patients who survive to hospital, improved imaging techniques and the advent of stent grafting has revolutionised diagnosis and management. Despite these modern techniques, only $15 \%$ of patients in this series survived. Improvement in outcomes for patients who survive to hospital is being actively sought and questions persist regarding the indications, timing, technical aspects and peri-operative management of patients undergoing endovascular repair for thoracic aortic injury. Perhaps, even more importantly, for this relatively young patient cohort, long-term outcomes and optimal surveillance patterns are not yet clear.

The Society for Vascular Surgery grading system has been used to determine timing of aortic repair. There is mounting evidence that conservative management is appropriate

Zsolt J. Balogh

zsolt.balogh@health.nsw.gov.au

1 Department of Traumatology, John Hunter Hospital and University of Newcastle, Newcastle, NSW, Australia in stable intimal lesions (Grade I) and that deferring repair of relatively stable (Grade II and III) lesions beyond the resuscitation phase of a patient's admission is associated with improved outcomes. Unfortunately, there is no clear consensus on what constitutes a "stable" lesion.

Payne and colleagues highlighted the difficulties in assessing severity of blunt aortic injury on CT and in identifying the need to prioritise aortic repair in the multiply injured patient (2). They grouped patients with total or partial transection or active contrast extravasation (SVS Grade IV) together with patients with other high-risk features such as left pleural effusion to define a severe injury. Radiologically Severe Injury was associated with increased aortarelated mortality, but not overall mortality. In this series, some patients with aortic pseudoaneurysm (Grade III) without suspicious features were considered not to have a severe injury and that group had survival of $>80 \%$. Thus, the timing and management of this intermediate grade injury remains controversial. It is worth highlighting that, outcome in the polytrauma patient with thoracic aortic injury is still predicted by established factors such as haemodynamic instability, high injury severity score and vasopressor requirement for hypotension.

Numerous technical aspects of endovascular repair also remain controversial; among them, the site and length of an adequate landing zone and the implications of covering the left subclavian artery to extend the landing zone (3). Son et al. retrospectively analysed the outcomes of TEVAR in their institution with $100 \%$ procedural success rate and a mean of almost 4 year follow-up (4). In this cohort, aortic dilatation at follow-up and reintervention rates did not change between the group with the recommended $20 \mathrm{~mm}$ proximal landing zone and those with shorter landing zones, suggesting that shorter landing zones may be acceptable in traumatic lesions compared to aneurysm disease. The inclusion of low-grade lesions in the operative series may have biased their results. In nine patients, the left subclavian artery was covered to extend the landing zone; no patients developed ischaemic symptoms or posterior circulation strokes. 
Endovascular techniques are also being progressively adopted for management of injury to junctional vessels including the subclavian artery. Injury to the subclavian artery is an uncommon but potentially a fatal injury. The series published by Kou of 22 such injuries highlights the difficulties inherent in a rare but life-threatening injury (5), not only the challenge of diagnosis (particularly in blunt injury) but also the knowledge vacuum that exists around the optimal management of these lesions particularly with the relatively recent adoption of endovascular techniques. The authors noted that only $27.3 \%$ of their patients with subclavian injury had hard signs of vascular injury affecting their upper limb; not surprisingly GCS $<12$, abbreviated injury scale of the head $>3$ and the presence of clavicular or scapular fractures were found to be risk factors for subclavian injury. They concluded that both open and endovascular techniques are effective in the management of these patients but that further investigation of the relative benefits is needed.

From this small sample of papers, it can be seen that, more data are required to accurately determine that answers to the questions that surround the diagnosis, investigation and management of major vascular trauma. Given the relative rarity of these injuries, large multicentre registries and trials will be needed as small, single-centre case series obtained over long periods of time will be obsolete in this rapidly changing field. Importantly, the questions of repair longevity and appropriate follow-up need to be answered for this young cohort of patients.

\section{Compliance with ethcial standards}

Conflict of interest The authors declare that they have no conflict of interest.

\section{References}

1. Trlica J, Kucěrová Š, Kočová E, Koči J, Habal P, Raupach J, Guňka I, Nechvátal L, Páral J, Šimek J, Šmejkal K, Frank M, Dědek T. Deceleration thoracic aortic ruptures in trauma center level I areas: a 6-year retrospective study. Eur J Trauma Emerg Surg. 2019. https://doi.org/10.1007/s00068-018-01063-4.

2. Payne RE, Nygaard RM, Fernandes JD, Sahgal P, Richardson CJ, Bashir M, Parekh K, Vardas PN, Suzuki Y, Corvera J, Krook JC, Calcaterra D. Blunt aortic injuries in the new era: radiologic findings and polytrauma risk assessment dictates management strategy. Eur J Trauma Emerg Surg. 2019. https://doi.org/10.1007/ s00068-019-01163-9.

3. Uchida K, Nishimura T, Yamamoto H, Mizobata Y. Efficacy and safety of TEVAR with debranching technique for blunt traumatic aortic injury in patients with severe multiple trauma. Eur J Trauma Emerg Surg. 2019. https://doi.org/10.1007/s00068-019-01123-3.

4. Son S, Jung H, Cho J, Oh T, Do YW, Lim KH, Kim G. Mid-term outcomes of endovascular repair for traumatic thoracic aortic injury: a single-center experience. Eur J Trauma Emerg Surg. 2019. https://doi.org/10.1007/s00068-019-01166-6.

5. Kou H, Liao C, Huang J, Hsu C, Wang S, Yang C, Kang S, Hsu Y, Hsieh C, Kuo I. Eighteen years' experience of traumatic subclavian vascular injury in a tertiary referral trauma center. Eur J Trauma Emerg Surg. 2019. https://doi.org/10.1007/s00068-01801070-5. 